\title{
Acoustic Material Characterization of Additively Manufactured Components
}

\author{
Leander Claes ${ }^{1}$, Axel Jäger², Sarah Johannesmann'1, Manuel Webersen¹, Mario Kupnik², \\ Bernd Henning ${ }^{1}$ \\ ${ }^{1}$ Measurement Engineering Group, Paderborn University, Warburger Str. 100, \\ 33098 Paderborn, Germany, \\ claes@emt.uni-paderborn.de \\ 2 Measurement and Sensor Technology, Technische Universität Darmstadt, Merckstraße 25, \\ 64283 Darmstadt, Germany
}

\begin{abstract}
:
Additively manufactured or $3 \mathrm{~d}$ printed components are commonly used for rapid prototyping or small series production purposes. Their mechanical properties, especially in the acoustic regime, are sparsely researched as of now. Therefore, we present a non-destructive measurement procedure to characterize the properties of additively manufactured components. We manufacture $3 \mathrm{~mm}$ thick plates by fused deposition modeling of polylactic acid (PLA). Using focused laser radiation, broadband ultrasonic Lambwaves are excited in the plates via the photoacoustic effect. These waves are detected using a purposebuilt ultrasonic transducer for plate waves. The measurement signals processed are compared to the output of a plate waveguide model. In an inverse procedure, the material parameters of the model are optimized to yield estimates for the material properties of the sample. Using this approach, we analyze the influence of printing process parameters on the mechanic and acoustic properties of the manufactured specimens.
\end{abstract}

Key words: additive manufacturing, material properties, acoustic characterization, non-destructive

\section{Introduction}

Additive manufacturing has proven to be a viable method for small series production. What hinders the use of additively manufactured components in critical applications, however, is the lack of a reliable method for testing and monitoring the quality of the manufacturing process.

Ultrasonic transmission measurements are a suitable method for non-destructive defect detection and condition monitoring. As acoustic waves, travelling through a specimen are influenced by the material's properties methods can be utilized to determine the properties of materials based on ultrasonic transmission measurements. These measurements are not limited to classical acoustic parameters of the material, such as longitudinal and transversal wave velocity, but also include mechanical properties, such as Young's modulus and Poisson's ratio.

In past publications, we present procedures to measure the material properties of metals [1] and polymers [2, 3]. In [4] we show that the procedure is applicable to highly anisotropic materials, such as fiber-reinforced plastics.
We adapt the inverse procedure to be used for the determination of effective materials properties of additively manufactured plates of polylactic acid (PLA).

\section{Measurement setup}

The instrumental setup applied for the measurement is similar to the one presented in [2] and [4]. As we are to analyze ultrasonic Lamb waves, the specimens need to be in shape of a plate. Due to the specimens being manufactured by fused deposition modelling, this is easily realized. However, common fused deposition modelling processes tend to create hollow structures with, for example, a honeycomb structure inside, to reduce the amount of filament used. For the presented method of analysis, we need the specimens to be manufactured as a solid plate without cavities. We aim to describe the material as a continuum without macroscopic structures.

In the experimental setup, (figure 1) ultrasonic Lamb waves are excited using focused, pulsed laser radiation. A nitrogen laser (LTB Lasertechnik Berlin, model MNL 103-PD High Power) 
emits the required laser pulses with a duration of $3 \mathrm{~ns}$ (full duration at half maximum) and an energy of $225 \mu \mathrm{J}$. Using a surface mirror and a cylindrical lens, the laser radiation is focused on a line on the specimen's surface. As nitrogen lasers emit radiation in the near ultraviolet regime, the lens is required to be made of fused quartz to avoid attenuation of the radiation. Mirror and lens are mounted on a linear actuator (Zaber Technologies Inc., model T-LSM) to vary the position of excitation of the Lamb waves. As the excitation is pulse-shaped (and thereby broadband), many Lamb waves are excited. These acoustic waves are detected by a purpose-built transducer, which is designed for the detection of plate waves. The active element of the transducer is a strip-shaped piezoceramic (PI Ceramic $\mathrm{GmbH}$, type PIC255). A detailed description of the construction of the transducer can be found in [2]. To ensure a good transmission of acoustic waves to the transducer, a thin layer of a coupling agent (GE, type multi-range coupling paste ZGT) is applied. A broadband charge amplifier (FEMTO Messtechnik $\mathrm{GmbH}$, model HQA-15M-10T) is used to increase the output signal strength of the transducer. Signals are recorded simultaneously to the radiation pulses of the laser using a USBoscilloscope (TiePie engineering, model Handyscope HS5-540).

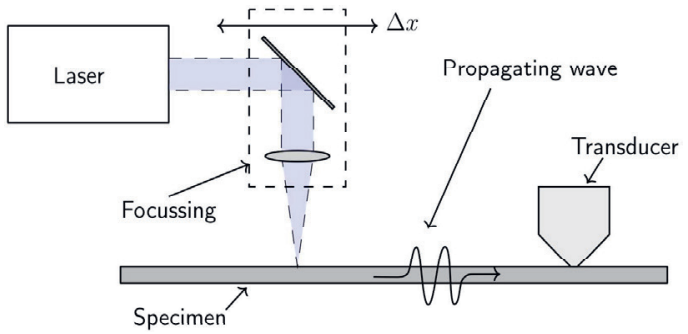

Fig. 1: Schematic of the measurement setup applied to excite and detect ultrasonic Lamb waves in plateshaped $3 d$ printed specimens.

\section{Manufacturing of the $\mathbf{3 d}$ printed samples}

All samples are manufactured using the method of Fused Deposition Modeling (FDM) [5]. Two different types of low-cost desktop 3D-printers are used: One Ultimaker 2+ (UM2+) and one Ultimaker 3 (UM3) by Ultimaker B.V., Geldermalsen, The Netherlands. Both printers share a nozzle diameter of $0.4 \mathrm{~mm}$ and are preconfigured to deposit material in layers with $0.1 \mathrm{~mm}$ thickness. The recommended material deposition profiles for PLA for both printers vary slightly (Tab. 1). Manually overwriting of the material profiles is possible and was done for one sample on the UM3 using a temperature of $220^{\circ} \mathrm{C}$. A regular white colored PLA blend (DasFilament, Braunschweig) is used for all samples.
Tab. 1: Material deposition profiles for PLA of both printers.

\begin{tabular}{|c|c|c|}
\hline & Speed & Temperature \\
\hline \hline UM2+ & $60 \mathrm{~mm} / \mathrm{s}$ & $210^{\circ} \mathrm{C}$ \\
\hline UM3 & $70 \mathrm{~mm} / \mathrm{s}$ & $200^{\circ} \mathrm{C}$ \\
\hline
\end{tabular}

The geometry of the samples is a square-shaped plate with a dimension of $150 \mathrm{~mm}$ by $150 \mathrm{~mm}$ and a thickness of $3 \mathrm{~mm}$. To support a uniform cool down of the sample, and, thus, prevent warping of the sample, the corners are rounded with a radius of $10 \mathrm{~mm}$. As measurements are performed only in the center of the plate, this does not influence the results.

During manufacturing, the plate is oriented parallel to the build plate of the printer. This results in 30 layers of material deposited. The fabrication time is around $24 \mathrm{~h}$ for each sample.

Slicing and generating of the toolpaths is done using the software Cura, Version 2.3.1. The strategy used to fill the volume of each layer is a hatched pattern angled $45^{\circ}$ to the edges of the plate. The hatch direction is flipped every layer to bond the individual fibers together.

\section{Signal acquisition and processing}

With the above-described measurement setup, signals are recorded with varying distance between excitation and detection of the ultrasonic wave. The samples are placed in the setup at an angle of $45^{\circ}$, so that the wave propagation takes place along the fibers of half of the layers and orthogonal to the other half. Starting close to the transducer, the lasers focus and therefore the point of excitation of the ultrasonic Lamb waves is moved away from the transducer in equidistant steps. At each step a signal is recorded. The resulting signals are arranged in a two-dimensional array. In this array the rows are the recorded signals and the columns represent the excitation positions. The matrix therefore has a temporal and a spatial axis. Applying a two-dimensional Fouriertransform to this matrix yields a depiction with a wavenumber and a frequency axis [7]. All modes excited by the broadband excitation are visible in figure 2 as ridges. This depiction bears similarity with a dispersion diagram but consists of a twodimensional field rather than individual lines. For better distinction from a dispersion diagram, we call it a dispersion map. There are several ways to improve the quality of the dispersion map, most of them being analog to the methods applied to ordinary Fourier-transforms. As the resolution in the spatial regime is dependent on the increment with which the excitation is moved 
when recording signals, we increase the resolution of the wavenumber by applying zeropadding to the spatial axis. Further, we apply a Tukey window (tapered cosine) to the data before transforming it. We also found that signals measured with the excitation close to the transducer decrease the quality of the dispersion map (i.e. the visibility and distinguishability of the modes). This may be due to the propagating waves being more similar to free field waves close to the excitation and Lamb-modes only forming after the waves traveled a certain distance. We therefore use a minimum distance between the excitation and the transducer of about $20 \mathrm{~mm}$. The resulting dispersion map of a $3 \mathrm{~mm}$ thick, additively manufactured plate made of polylactic acid (PLA) is shown in figure 2 .

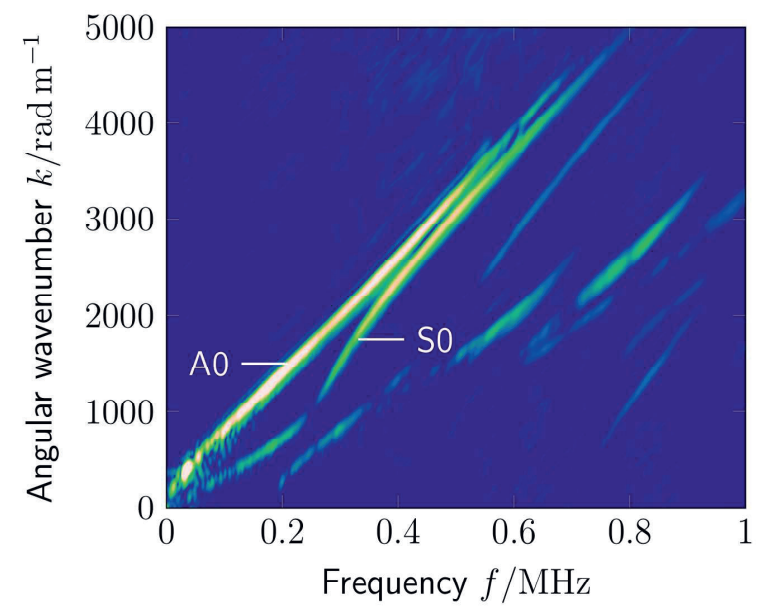

Fig. 2: Dispersion map of an additively manufactured PLA plate using the UM2+.

In the dispersion map, several modes are visible, with the $\mathrm{AO}$ - and S0-modes being most pronounced. Several higher order modes are visible as well, with most of them travelling at approximately the transversal wave velocity we expect of the material. The overall similarity of the dispersion map (although being incomplete) to the dispersion diagram of an isotropic plate waveguide suggests a mostly isotropic behavior of the material. This is quite remarkable as one might expect a more pronounced anisotropic behavior due to the manufacturing process.

\section{Material characterization}

We apply an inverse procedure as presented in [4], to further characterize the material properties of the PLA plate. As the optimization process to be used requires initial values, we begin by finding rough estimates for the parameters. For these estimates we first assume the material to be isotropic, with longitudinal and transversal wave velocities $\left(c_{\mathrm{L}}\right.$ and $\left.c_{\mathrm{T}}\right)$ assumed to be independent of the direction the wave is travelling. As there is a strong relation between the shape of the dispersion diagram of an isotropic acoustic waveguide and the material's longitudinal and transversal wave velocities, we can extract these from the dispersion map in figure 2. For our model requires the elastic properties of the material (i.e. Young's modulus $E$ and Poisson's ratio $v$ ) we calculate them from the estimated wave velocities using the following relations [8]:

$$
\begin{gathered}
E=\frac{\rho c_{\mathrm{T}}^{2}\left(3 c_{\mathrm{L}}^{2}-4 c_{\mathrm{T}}^{2}\right)}{c_{\mathrm{L}}^{2}-c_{\mathrm{T}}^{2}}, \\
v=\frac{1-2\left(\frac{c_{\mathrm{T}}}{c_{\mathrm{L}}}\right)^{2}}{2-2\left(\frac{c_{\mathrm{T}}}{c_{\mathrm{L}}}\right)^{2}} .
\end{gathered}
$$

We use a waveguide model based on a semianalytical finite element (SAFE) approach to calculate a dispersion diagram for a comparison with the measurement results. For the waveguide model, we assume one spatial direction of the plate to be extended infinitely and apply an analytic formulation of wave propagation in the other direction [6, 10]. It follows that the mesh of the simulation is only one-dimensional (in thickness-direction of the plate), resulting in a short computation time for the dispersion diagram. For a detailed description of the model applied, please refer to [4].

The optimization process requires a cost function to compare simulation and measurement. Ideally, the cost function has a single minimum for the optimization algorithm to find. Establishing the cost function can prove challenging for complex comparison problems such as this. We therefore prefer a straightforward approach to compare our twodimensional dispersion maps to the frequencywavenumber-pairs calculated by the waveguide model: We extract the values from the measured dispersion map where the points of the dispersion diagram are located. We then sum up all these values, divide by the number of points calculated in the dispersion diagram. This alone would result in high values if the simulated dispersion diagram and measured dispersion map match. As most optimization algorithms are implemented in a way so the find minima, we invert the calculated value.

We apply the Nelder-Mead simplex algorithm [9] to the cost function with the material properties of the model as parameters to optimize. When simulated dispersion diagram and the dispersion map generated from the measured signals match, we assume the model parameters to be an estimation for the specimen's material parameters. Because of the manufacturing process and the resulting inner structure of the 
specimen, we assume a transversely isotropic material model, resulting in four material parameters to optimize: Young's modulus parallel and orthogonal to the plates surfaces $E_{\mathrm{P}}$ and $E_{\mathrm{O}}$, and the corresponding Poisson's ratios $v_{\mathrm{P}}$ and $v_{\mathrm{O}}$. The density $\rho$ and the thickness of the plate are measured directly and excluded from the optimization process.

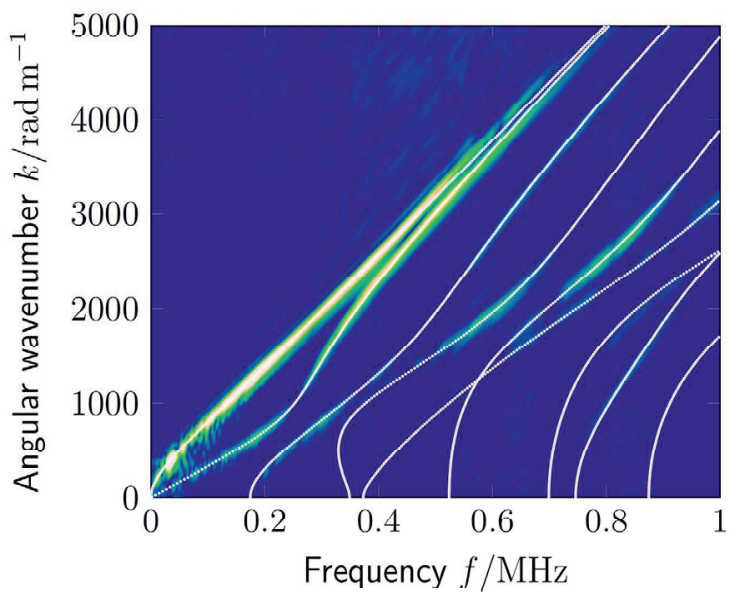

Fig. 3: Dispersion map of an additively manufactured PLA plate (UM2+) superimposed with a dispersion diagram generated by simulation after the optimization process.

To verify the result of the optimization process, simulation and measurement result are superimposed (figure 3). All modes are matched well between measurement and simulation with a transversely isotropic material. The resulting estimations for the material parameters of the sample manufactured using the UM2+ are:

$$
\begin{gathered}
E_{\mathrm{P}}=4.12 \mathrm{GPa} \\
E_{\mathrm{O}}=4.14 \mathrm{GPa} \\
v_{\mathrm{P}}=0.341 \\
v_{\mathrm{O}}=0.343
\end{gathered}
$$

The density $\rho$ is measured directly $\left(1231 \mathrm{~kg} / \mathrm{m}^{3}\right)$, the plate's thickness is, as mentioned before $3 \mathrm{~mm}$. The material properties can be expressed as wave velocities parallel and orthogonal to the plates surface as well $[8,13]$ :

$$
\begin{aligned}
& c_{\mathrm{L}, \mathrm{P}}=2254 \mathrm{~m} / \mathrm{s} \\
& c_{\mathrm{T}, \mathrm{P}}=1106 \mathrm{~m} / \mathrm{s} \\
& c_{\mathrm{L}, \mathrm{O}}=2261 \mathrm{~m} / \mathrm{s} \\
& c_{\mathrm{T}, \mathrm{O}}=1111 \mathrm{~m} / \mathrm{s}
\end{aligned}
$$

These results support our previous assumption that the behavior of the plate is mostly isotropic. As measurements are conducted with mainly ultrasonic waves with frequencies up to $1 \mathrm{MHz}$, the resulting material parameters can only be considered valid in this regime. Especially the results for Young's modulus and Poisson's ratio are not generally the same for ultrasonic measurements and static experiments such as destructive tensile tests. However, [11] states Young's modulus of bulk polylactic acid to be $3.5 \mathrm{GPa}$ (measured by tensile test), which is fairly close to the result in this publication given the multitude differences in the acquisition of the value (acoustic vs. tensile measurement, printed vs. bulk material, different manufacturer, etc.). The most probable cause for our measurement deviation upwards is due to the frequency dependence of the material properties [3]. It typically leads to an increase of Young's modulus with increasing frequency (tensile test being considered a quasi-static measurement).

\section{Influence of manufacturing process parameters}

As our goal is to analyze the influence of the manufacturing process parameters on the resulting material properties, we examine three different samples: One is manufactured using the Ultimaker $2+(\mathrm{UM} 2+)$ at a process temperature of $210^{\circ} \mathrm{C}$ and a deposition speed of $60 \mathrm{~mm} / \mathrm{s}$. This sample is the one analyzed in the previous section. The other two samples are manufactured using the Ultimaker 3 with a deposition speed of $70 \mathrm{~mm} / \mathrm{s}$. They differ only in the processing temperature, which is $200^{\circ} \mathrm{C}$ for sample number two and $220^{\circ} \mathrm{C}$ for sample number three.

Tab. 2: Comparison of measured material properties for samples with manufacturing process parameters.

\begin{tabular}{|c|c|c|c|}
\hline Sample & 1 & 2 & 3 \\
\hline \hline Printer & UM2+ & UM3 & UM3 \\
\hline Temp. & $210^{\circ} \mathrm{C}$ & $200{ }^{\circ} \mathrm{C}$ & $220^{\circ} \mathrm{C}$ \\
\hline Speed & $60 \mathrm{~mm} / \mathrm{s}$ & $70 \mathrm{~mm} / \mathrm{s}$ & $70 \mathrm{~mm} / \mathrm{s}$ \\
\hline \hline$E_{\mathrm{P}}$ & $4.12 \mathrm{GPa}$ & $4.10 \mathrm{GPa}$ & $3.99 \mathrm{GPa}$ \\
\hline$E_{\mathrm{O}}$ & $4.14 \mathrm{GPa}$ & $3.58 \mathrm{GPa}$ & $3.98 \mathrm{GPa}$ \\
\hline$v_{\mathrm{P}}$ & 0.341 & 0.200 & 0.245 \\
\hline$v_{\mathrm{O}}$ & 0.343 & 0.364 & 0.341 \\
\hline$c_{\mathrm{L}, \mathrm{P}}$ & $2254 \mathrm{~m} / \mathrm{s}$ & $2191 \mathrm{~m} / \mathrm{s}$ & $2091 \mathrm{~m} / \mathrm{s}$ \\
\hline$c_{\mathrm{T}, \mathrm{P}}$ & $1111 \mathrm{~m} / \mathrm{s}$ & $1064 \mathrm{~m} / \mathrm{s}$ & $1128 \mathrm{~m} / \mathrm{s}$ \\
\hline$c_{\mathrm{L}, \mathrm{O}}$ & $2261 \mathrm{~m} / \mathrm{s}$ & $2179 \mathrm{~m} / \mathrm{s}$ & $2148 \mathrm{~m} / \mathrm{s}$ \\
\hline$c_{\mathrm{T}, \mathrm{O}}$ & $1106 \mathrm{~m} / \mathrm{s}$ & $1186 \mathrm{~m} / \mathrm{s}$ & $1135 \mathrm{~m} / \mathrm{s}$ \\
\hline \multicolumn{2}{|r}{}
\end{tabular}

There are several aspects to be noted when comparing the results for all three samples. Most obvious is a big difference in Poisson's ratio parallel to the plate's surface $v_{\mathrm{P}}$ when comparing samples two and three to sample one. One might assume that, as sample one is manufactured 
with a smaller deposition speed, decreasing the deposition speed increases $v_{\mathrm{p}}$. When observing Young's Modulus both in parallel and orthogonal to the plate's surface, samples one and three suggest a more isotropic behavior than sample two. As sample two and three are manufactured with the same process parameters besides the temperature, we can conclude that increasing the temperature results in more isotropic properties of the manufactured component. The wave velocities reflect this behavior as well. The slight decrease in longitudinal wave velocity in sample three could suggest a decrease in molecular length and thereby an aging of the applied polymer [12] due to the higher process temperature.

The observed change in material properties resulting from modified process parameters seem plausible and match the expectations of the authors. Both a higher temperature and a lower deposition speed seem to increase the adhesion between the deposited layers and lead to a material that behaves increasingly similar to an isotropic solid in the considered frequency range. The deposition speed seems to have a bigger impact on Poisson's ratio, while the temperature influences Young's modulus. As sample one already shows nearly isotropic behavior, these parameters should be applicable if one aims to create an isotropic component.

\section{Conclusion and outlook}

Using thermoelastically excited ultrasonic waves, the material properties of additively manufactured samples have been determined. The measurement method was further used to exemplarily show the influence of the deposition process parameters to the material properties of the created components.

The possibility to non-destructively determine acoustic and mechanical material properties paves the way for many applications and further research. With regards to the measurement method the authors aim to expand it to yield data for absorption using more complex material models [3]. Applying the presented measurement procedure, the influence of further process parameters can be observed and analyzed. The procedure can thus be applied to compare the results of different printer models and printing methods. It allows for an optimization of the result of the printing process with regards to the material parameters. With the ability to determine the acoustic material parameters of additively manufactured components, these components can now be used in the design of acoustic applications. These design processes are usually supported by simulations. The acquired material data can be used to realistically simulate the behavior of the components. Influencing material properties by varying the deposition process parameters also allows to create components where specific parameters show a gradient. These functionally graded materials can for example be used for acoustic matching layers or absorbers.

\section{References}

[1] L. Claes, F. Bause, J. Rautenberg, and B. Henning, Detection of ultrasonic plate waves using ceramic strip transducers, Sensor Cenferences Nürnberg, 19-21 (2015); doi: 10.5162/sensor2015/P3.3

[2] L. Claes, T. Meyer, F. Bause, J. Rautenberg, and B. Henning, Determination of the material properties of polymers using laser-generated broadband ultrasound, Journal of Sensors and Sensor Systems, 5 (1), 187-196 (2016) doi: 10.5194/jsss-5-187-2016.

[3] F. Bause, J. Rautenberg, N. Feldmann, M. Webersen, L. Claes, H. Gravenkamp, and B. Henning, Ultrasonic transmission measurements in the characterization of viscoelasticity utilizing polymeric waveguides, Measurement Science and Technology, 27 (10), 105601 (2016); doi: $10.1088 / 0957-0233 / 27 / 10 / 105601$

[4] M. Webersen, S. Johannesmann, L. Claes, and B. Henning, Characterization of continuous-fiber reinforced thermoplastics using thermoacoustically excited ultrasonic Lamb waves, Ultrasonics Symposium (IUS), 2016 IEEE International, 1-4 (2016); doi: 10.1109/ULTSYM.2016.7728585

[5] S. Scott Crump, Apparatus and method for creating three-dimensional objects, US PAT 5121329 A

[6] I. Bartoli, A. Marzani, F. Di Lanza Scalea, and E. Viola, Modeling wave propagation in damped waveguides of arbitrary cross-section, Journal of Sound and Vibration 295 (3-5), 685-707 (2006); doi: 10.1016/j.jsv.2006.01.021

[7] D.N. Alleyne, P. Cawley, A 2-dimensional Fourier transform method for the quantitative measurement of Lamb modes, Ultrasonics Symposium IEEE 1990, 1143-1146 (1990); doi: 10.1109/ULTSYM.1990.171541

[8] R. Lerch, G.M. Sessler, D. Wolf, Technische Akustik (2009); doi: 10.1007/978-3-540-49833-9

[9] J.-A. Nelder R. Mead, A simplex method for function minimization, The Computer Journal, 7, 308-313 (1965); doi: 10.1093/comjnl/7.4.308

[10] T. Hayashi, W.-J. Song, and J. L. Rose, Guided wave dispersion curves for a bar with an arbitrary cross-section, a rod and rail example, Ultrasonics, 41 (3), 175-183 (2003); doi: 10.1016/S0041-624X(03)00097-0

[11] M. Jamshidian, E.A. Tehrany, M. Imran, M. Jacquot, S. Desobry, Poly-Lactic Acid. Production, Applications, Nanocomposites, and 
Release Studies, Comprehensive Reviews in Food Science and Food Safety, 9 (5), 552-571 (2010); doi: 10.1111/j.1541-4337.2010.00126.x

[12] M. Webersen, M. Hüttner, F. Bause, E. Moritzer, B. Henning, Zerstörungsfreie Charakterisierung des hydrothermischen Alterungsverhaltens von Polymeren, 18. GMA/ITG-Fachtagung Sensoren und Messsysteme, 683-688 (2016); doi: 10.5162/sensoren2016/P6.4

[13] J. Rautenberg, Ein wellenleiterbasiertes Verfahren zur Bestimmung von Materialdaten für die realitätsnahe Simulation von Schallausbreitungsphänomenen am Beispiel stark absorbierender Kunststoffe, Dissertation, Universität Paderborn (2012) 